\title{
Description of the fusion-fission reactions in the framework of dinuclear system conception
}

\author{
Sh. A. Kalandarov ${ }^{1,2}$, G. G. Adamian ${ }^{1}$, N. V. Antonenko ${ }^{1}$ and \\ J.P. WIELECZKO ${ }^{3}$
}

1 Joint Institute for Nuclear Research, 141980 Dubna, Russia

${ }^{2}$ Institute of Nuclear Physics,702132 Tashkent, Uzbekistan

3 GANIL, CEA et IN2P3-CNRS, B.P. 55027, F-14076, Caen Cedex, France

\begin{abstract}
Within the dinuclear system model fusion-fission reactions ${ }^{78} \mathrm{Kr}+{ }^{40} \mathrm{Ca}$ and ${ }^{86} \mathrm{Kr}+{ }^{48} \mathrm{Ca}$ is investigated. The charge distributions of the decay products are predicted at bombarding energy 10 $\mathrm{MeV} /$ nucleon. The competition is treated between complete fusion followed by the decay of compound nucleus and quasifission channels. The possible explanation of the odd-even staggering in the yield of the final reaction products at high excitation energies is discussed.
\end{abstract}

\section{Introduction}

The experimentally measured charge, mass, and isotopic distributions of reaction products carry important information about both the reaction mechanism and the interfacing structure of nuclei and reaction dynamics [1-7]. One of such interesting experimental findings in the binary heavy-ion reactions is the persistence of the odd-even staggering in the charge distributions of reaction products at relatively high bombarding energies $[3,5,6]$. The oddeven staggering effect was found to be smaller for neutron-rich system than for the neutron-deficient one $[2,5]$. One of the possible explanations for the odd-even staggering at high excitation energies is that the fragments with 
$5 \leq Z \leq 16$ mainly come out at the first step of de-excitation cascade and these nuclei preserve their structure during the formation stage and, thus, the primary charge distribution has strong odd-even staggering. The further de-excitation of these fragments slightly decreases the amplitude of the odd-even staggering.

The purpose of the present work is to demonstrate the influence of isospin of the system on the reaction mechanism and, correspondingly, on the charge, mass, isotopic distributions of the reaction products and on the odd-even staggering effect.

The detailed theoretical study will be carried out within the dinuclear system (DNS) model [7]. In this model the cluster emission is treated under the assumption that the complex fragments are produced by a collective motion of the nuclear system in the charge (mass) asymmetry coordinate with further thermal escape over the Coulomb barrier. The emission barriers for complex fragments are calculated within the DNS model by using the double-folding procedure (with the Skyrme-type density-depending effective nucleon-nucleon interaction) for the nuclear part of the nucleus-nucleus interaction potential. Both the evaporation and binary decay are treated in the same way. The correct definition of the emission barriers and of their dependence on the angular momentum allows us to calculate the charge, mass, and kinetic energy distributions of the emitted complex fragments. The main ingredients of our description is the sophisticated potential energy as a function of angular momentum. In our case the maximum possible angular momentum in the system is not adjustable parameter. It is calculated within our model by using the nucleus-nucleus interaction potential [7]. The dynamics plays a role at high angular momenta when the quasifission becomes important contributing to the yields of fission-like fragments. Note that the high angular momenta have larger contribution to the cross section.

The DNS model was applied to predict the charge and mass distributions of the products in the reactions ${ }^{78,82} \mathrm{Kr}+{ }^{40} \mathrm{Ca}$ at the incident energy 5.5 $\mathrm{MeV} /$ nucleon [2]. Our results were in a good agreement with experimental data slightly underestimating the cross sections near the entrance channel, where the contributions from the deep-inelastic reaction channel may be present in the experimental data. Here we apply the well tested model to predicts the yields of products in the binary reactions ${ }^{78} \mathrm{Kr}+{ }^{40} \mathrm{Ca}$ and ${ }^{86} \mathrm{Kr}+{ }^{48} \mathrm{Ca}$ at bombarding energy $10 \mathrm{MeV} /$ nucleon. For these reactions, the ISODEC experiments have been done at the INFN-Laboratori Nazionali del Sud by using the CHIMERA device. Some preliminary experimental results were published in Ref. [4]. 


\section{DNS model}

The DNS model $[7,8]$ describes an evolution of the charge and mass asymmetry degrees of freedom, which are defined here by the charge and mass (neutron) numbers $Z$ and $A(N=A-Z)$ of light nucleus of the DNS. According to this description, there are nucleon drift and nucleon diffusion between the DNS nuclei and eventually either the CN is formed (the complete fusion) or the DNS with given $Z$ and $A$ is formed and decays (quasifission). After the formation, the excited $\mathrm{CN}$ decays by various channels including again the formation of certain DNS and their decay. The CN formation and its consequent decay are not necessarily the ultimate results of the evolution of the initial DNS. In addition to the contributions from the $\mathrm{CN}$ decay, the binary decay component is related to the quasifission mechanism. The competition between the complete fusion and quasifission depends on the value of maximum angular momentum deposited in the system. The quasifission and $\mathrm{CN}$ decays are hardly distinguished in the experiments because in both cases two fragments are produced by the decay of the DNS formed during the diffusion process in the mass (charge) asymmetry coordinate with and without stage of the $\mathrm{CN}$ formation.

The production cross section of nucleus with charge $Z$ and mass $A$ numbers is calculated as follows [7]

$$
\sigma_{Z, A}\left(E_{\text {c.m. }}\right)=\sum_{J=0}^{J_{\max }^{*}} \sigma_{Z, A}\left(E_{\text {c.m. }}, J\right)=\sum_{J=0}^{J_{\text {max }}^{*}} \sigma_{c a p}\left(E_{\text {c.m. }}, J\right) W_{Z, A}\left(E_{\text {c.m. }}, J\right)
$$

where $\sigma_{c a p}$ is the partial capture cross section which defines the transition of the colliding nuclei over the Coulomb barrier and the formation of the initial DNS and $E_{\text {c.m. }}$ is the kinetic energy. This transition probability is calculated with the Hill-Wheeler formula. The value of $W_{Z, A}$ is the formation-decay probability of the DNS with a given asymmetries $Z$ and $A$. The probability of the DNS formation is calculated statistically by using the stationary solution of the master equation with respect to the charge and mass asymmetries and depends on the potential energy of the DNS configurations at touching distance and on the thermodynamical temperature. The probability of the DNS decay in the relative distance $R$ is calculated by using the transition state method. This probability depends on the difference between the potential energies of the DNS configurations at the touching distance and at the barrier position. The maximum angular momentum $J_{\max }^{*}=J_{\max }+\delta J_{\max }$ which leads to the capture without the pre-equilibrium decay is defined here by taking into account the effect of 
pre-equilibrium emission. Here, the value of $J_{\max }$ is limited by either the kinematical angular momentum $J_{\text {kin }}=\left[2 \mu\left(E_{\text {c.m. }}-V\left(R_{b}\right)\right) / \hbar^{2}\right]^{1 / 2} R_{b}\left(R_{b}\right.$ is the position of the Coulomb barrier with the height $V\left(R_{b}\right)$ and $\mu$ is the reduced mass) or by the calculated critical angular momentum $J_{c r}$ depending on which one is smaller, $J_{\max }=\min \left[J_{k i n}, J_{c r}\right]$, and $\delta J_{\max }$ is the average angular momentum which is taken away from the system by pre-equilibrium particles (Table 1). For the case of partial wave $J[J>\delta J]$, we calculate the partial formation-decay probability $W_{Z, A}$ and the partial capture cross section at $J-\delta J$ and $J$, respectively. It should be noted that the calculated capture cross sections with this method are in a good agreement with those obtained with the dynamical model [9]. The excitation energy of the system after the pre-equilibrium emission is decreased by the $\delta E$ value according to the Table 1. Finally, after each pre-equilibrium emission we calculate the evolution in mass asymmetry degree of freedom and decay of the DNS. The details of calculations of $\sigma_{c a p}, W_{Z, A}$, and, correspondingly, $\sigma_{Z, A}\left(E_{\text {c.m. }}\right)$ are given in Ref. $[7,8]$.

\section{Results and discussions}

We calculate the charge distributions of the products in the ${ }^{86} \mathrm{Kr}+{ }^{48} \mathrm{Ca}$ reaction at bombarding energy $E_{\text {lab }}=10 \mathrm{MeV} /$ nucleon and compared them with the charge and mass distributions in the neutron-deficient ${ }^{78} \mathrm{Kr}+{ }^{40} \mathrm{Ca}$ reaction at the same bombarding energy. The calculated maximum angular momenta and $\mathrm{CN}$ excitation energies (at $J=0$ ) involved in the ${ }^{86} \mathrm{Kr}+{ }^{48} \mathrm{Ca}$ and ${ }^{78} \mathrm{Kr}+{ }^{40} \mathrm{Ca}$ reactions are $J_{\max }=90, E_{C N}^{*}=270 \mathrm{MeV}$ and $J_{\max }=73, E_{C N}^{*}=217$ $\mathrm{MeV}$, respectively. The driving potentials (normalized to the energies of the corresponding rotating $\mathrm{CN}$ ), which are responsible for the formation of different DNS configurations, are shown in Fig. 1 for both reactions. Comparing these driving potentials, one can conclude that the odd-even staggering decreases with increasing $N / Z$ ratio in the system. The main reason for this is that the pairing energy decreases with increasing mass number of the nucleus. The pairing energies and shell corrections of the DNS nuclei are included into the driving potential through their binding energies. So, the staggering effects in the yields of final products are related to the corresponding structure effects in the nuclear binding energies [10].

One can see that for the neutron-deficient system the potential energy is lower than for neutron-rich system at the same value of angular momentum (the value of $B_{1}+B_{2}-B_{12}$ increases faster than $V\left(R_{m}, Z, A, J\right)$ decreases with increasing total neutron number). This means that the formation of 

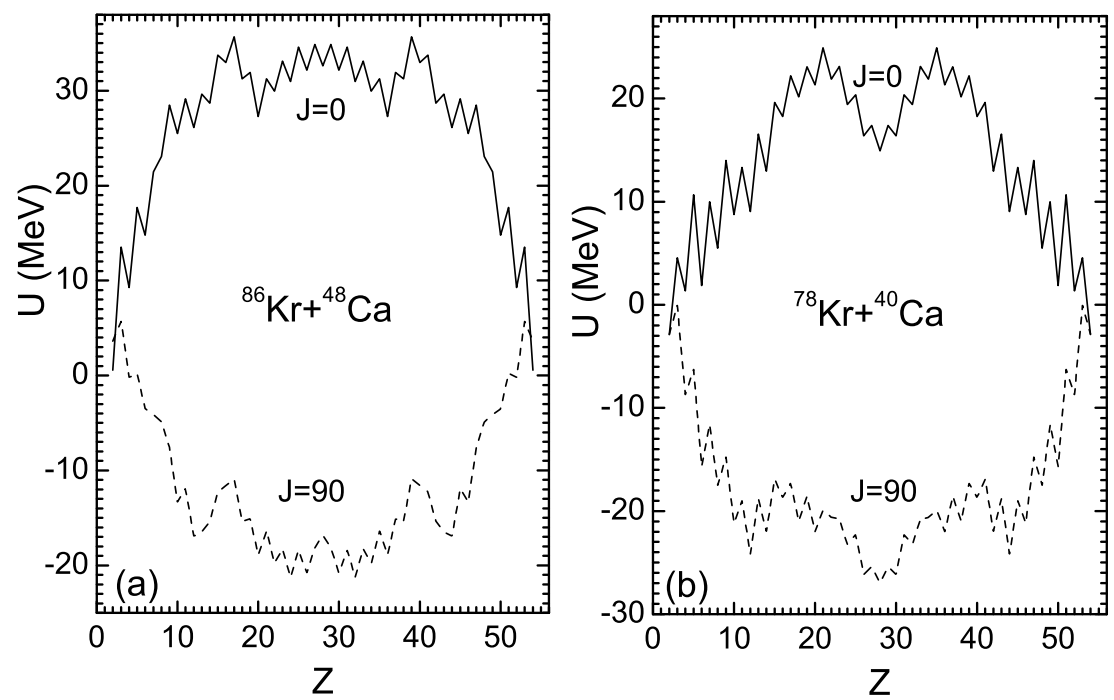

Figure 1: The driving potentials at angular momenta $J=0$ and 90 for the systems ${ }^{86} \mathrm{Kr}+{ }^{48} \mathrm{Ca}$ (a) and ${ }^{78} \mathrm{Kr}+{ }^{40} \mathrm{Ca}(\mathrm{b})$. The value of $U$ is normalized to the energy of the rotating $\mathrm{CN}$. The value $A$ is related to $Z$ to supply the minimum of $U$.

the DNS configurations is more probable for the neutron-deficient system. So, one can expect the larger yield of fission-like fragments in the neutrondeficient system than in the neutron-rich one. With increasing angular momentum the potential energy for symmetric DNS configurations becomes negative at $J>J_{0}=45$ for ${ }^{78} \mathrm{Kr}+{ }^{40} \mathrm{Ca}$ and at $J>J_{0}=70$ for ${ }^{86} \mathrm{Kr}+{ }^{48} \mathrm{Ca}$. At these large angular momenta the calculated driving potentials show global minimum at the symmetric DNS but not at the CN configuration and the charge (mass) drift pushes the system toward symmetry. The complete fusion becomes energetically denied and higher $J$ waves do not lead to fusion, but to the quasifission resulting in the fragments produced as the binary decay products of the transient DNS originated from the target-projectile DNS. This implies that at higher partial waves, most of the heavy complex fragments are produced by quasifission. At $J \approx J_{0}$ the $\mathrm{CN}$ and symmetric DNS potential energies coincide, and one can observe the coexistence of the quasifission and fusion-decay events. Thus, at the value of $J_{0}$ the reaction mechanisms become less clear-cut. At $J<J_{0}$ the driving potential is positive, the CN configuration is energetically favorable, and the decay products are mainly from the decay of the excited $\mathrm{CN}$.

In Fig. 2, we compare the charge distributions of the emitted complex fragments in the reactions ${ }^{78} \mathrm{Kr}(10 \mathrm{MeV} /$ nucleon $)+{ }^{40} \mathrm{Ca}$ and ${ }^{86} \mathrm{Kr}(10$ 
$\mathrm{MeV} /$ nucleon $)+{ }^{48} \mathrm{Ca}$. In both cases, the odd-even effects are present in the production cross sections

$$
\sigma_{Z}\left(E_{\text {c.m. }}\right)=\sum_{A} \sigma_{Z, A}\left(E_{\text {c.m. }}\right)
$$

of the fragments with $Z=2-12$. For heavier fragments, these effects are not visible because the odd-even structures of the charge distributions are washed out due to the sequential evaporation. So, the amplitude of the staggering depends on the deposited energy. The primary decay products with $Z \leq 12$ are less excited and emit mainly $\alpha$-particles. This retains the relatively strong odd-even staggering effect in the charge distributions for these nuclei. Thus, the reason why the odd-even staggering effect persists even at the intermediate energies $[5,6]$ becomes clear. The comparison of the predicted charge distributions with the preliminary experimental results for the reactions considered has already made in Ref. [4].
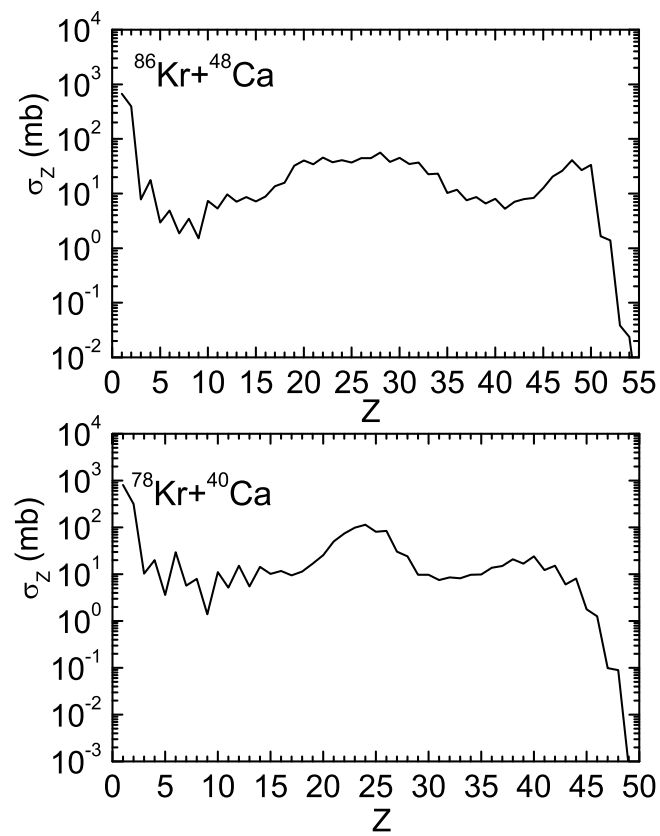

Figure 2: The calculated charge distributions of the products in the reactions ${ }^{86} \mathrm{Kr}+{ }^{48} \mathrm{Ca}$ and ${ }^{78} \mathrm{Kr}+{ }^{40} \mathrm{Ca}$ at bombarding energy $10 \mathrm{MeV} /$ nucleon.

As found in Ref. [11], the staggering depends in a more complicated way 
on the history of the evaporation chain than the schematic interpretation suggested in Ref. [10]. In Ref. [10], the staggering is presented in the isotopic yields at the last but one evaporation step through the pairing effect on nucleon separation energies.

In Fig. 2, the odd-even staggering is much smaller for the neutron-rich system comparing to the neutron-deficient one. The maxima of the yields of symmetric fragments are located at the same position for both system, but for the neutron-rich system the dependence on $Z$ is more flat. The evaporation residue charge distributions is shifted to smaller $Z$ values for the neutron-deficient system. It is interesting to see that the yields of the emitted light complex fragments are much smaller in the ${ }^{86} \mathrm{Kr}+{ }^{48} \mathrm{Ca}$ reaction. For example, the cross section for carbon emission is about 6 times smaller than in the ${ }^{78} \mathrm{Kr}+{ }^{40} \mathrm{Ca}$ reaction. This is mainly because of smaller formation probability of corresponding DNS configurations in the ${ }^{86} \mathrm{Kr}+{ }^{48} \mathrm{Ca}$ reaction comparing to the ${ }^{78} \mathrm{Kr}+{ }^{40} \mathrm{Ca}$ reaction.

\section{Conclusions}

We investigated the reactions ${ }^{78} \mathrm{Kr}+{ }^{40} \mathrm{Ca}$ and ${ }^{86} \mathrm{Kr}+{ }^{48} \mathrm{Ca}$ at bombarding energy $10 \mathrm{MeV} /$ nucleon within the DNS model. Our aim was to provide the predictions for these reactions. We found the persistence of odd-even staggering effects in the charge and mass distributions of the reaction products that is in agreement with the experimental observations of Refs. [5,6]. The charge distributions for the neutron-rich system are more flat as compared with the neutron-deficient system.

To elucidate the influence of neutron-excess of the initial system on the formation of final decay fragments, it would be better to perform the experiments at the incident energies about $4 \mathrm{MeV} /$ nucleon for the reactions considered.

\section{Acknowledgements}

This work was partially supported by RFBR and IN2P3-JINR Cooperation program.

\section{References}

[1] W.-U. Schröder and J.R. Huizenga, in Treatise on Heavy-Ion Science, edited by D.A. Bromley, Vol. 2 (Plenum Press, New York, 1984) p.115; 
V.V. Volkov, in Treatise on Heavy-Ion Science, edited D.A. Bromley, Vol. 8 (Plenum Press, New York, 1989) p.101.

[2] G. Ademard et al., Phys. Rev. C 83, 054619 (2011).

[3] I. Lombardo et al., Phys. Rev. C 84, 024613 (2011).

[4] S. Pirrone et al., Journal of Physics: Conf. Ser. 515, 012018 (2014).

[5] S. Piantelli et al., Phys. Rev. C 88, 064607 (2013).

[6] G. Casini et al., Phys. Rev. C 86, 011602(R) (2012).

[7] Sh. A. Kalandarov, G.G. Adamian, N.V. Antonenko, and W.Scheid, Phys. Rev. C 82, 044603 (2010); 83, 054611 (2011).

[8] V.V. Volkov, Izv. AN SSSR ser. fiz. 50, 1879 (1986).

[9] V.V. Sargsyan, G.G. Adamian, N.V. Antonenko, and W. Scheid, Eur. Phys. J. A 45, 125 (2010); V.V. Sargsyan, G.G. Adamian, N.V. Antonenko, W. Scheid, and H.Q. Zhang, Phys. Rev. C 84, 064614 (2011); 85, 024616 (2012); 91, 014613 (2015).

[10] M.V. Ricciardi, A.V. Ignatyuk, A. Kelic, P. Napolitani, F. Rejmund, K.-H. Schmidt, and O. Yordanov, Nucl. Phys. A733, 299 (2004).

[11] M. D’Agostino et al., Nucl. Phys. A861, 47 (2011). 